\title{
Pathologie digestive en zone rurale africaine
}

\author{
Pr. F. Klotz \\ C) Springer-Verlag France 2012
}

Loin du goudron, sur les méandres de la piste, les villages s'égrènent, se ressemblant comme des frères avec leurs concessions, leurs cases au toit de paille, leurs enclos enfermant ovins et caprins, leurs enfants s'égayant autour des marigots, les femmes occupées par le puits ou en train de piler le mil pour préparer le repas. Au gré des petits chemins $\mathrm{du}$ village on ne rencontre bien souvent pas de latrines auprès des cases. La pauvreté est prégnante.

Lors des rares consultations itinérantes les plaintes digestives sont fréquentes : diarrhées de l'enfant, dysenteries, rectorragies, douleurs abdominales en cadre ou épigastralgies, émission de vers dans les selles. L'examen est peu contributif, les moyens d'explorations sont inexistants, que ce soit la coprologie ou l'endoscopie.

Il faut donc se fier à son bon sens clinique et aux probabilités liées au contexte épidémiologique.

Dans la plupart des villages l'hygiène alimentaire n'est pas pratiquée, la notion du péril fécal non connue. De multiples maladies infectieuses transmissibles à tropisme digestif sont présentes de manière endémique ou épidémique. La danse des agents pathogènes est quotidienne impliquant : diarrhées aiguës frappant les enfants avec leur lot de déshydratation et de décès, helminthiases digestives transmises de manière itérative avec des taux d'infestation pouvant battre des records et leur lot d'accidents éventuels, mécaniques ou hématologiques. Cortège de bactéries de virulence variable : de l'escherichia coli entéropathogène aux shigellas en passant par les salmonellas d'expression clinique variable allant de la simple diarrhée au syndrome cholériforme ou à la dysenterie. Transmission intense par les mains sales d'amibes et autres protozoaires marqueurs du péril fécal. Ravage de la bilharziose intestinale chez ces enfants jouant quotidiennement au marigot avec diarrhée sanglante, hépatosplénomégalie, voire hémorragie digestive haute.

Devant ce cortège de signes digestifs fonctionnels, allant des douleurs abdominales aux troubles majeurs du transit, l'agent de santé responsable des ces villages agit à tâtons avec des moyens dérisoires, souvent inadaptés quant à leur vertu curative.

Avec peu de moyens, il faut quadriller ces régions en inculquant l'éducation sanitaire dès le plus jeune âge aux enfants à l'école primaire. Il faut que les mères comprennent ce qu'est le péril fécal et l'hygiène élémentaire ainsi que tout ce qui en découle pour la santé de leurs enfants. Il faut sans cesse répéter en formant agents de santé communautaire, instituteurs, infirmiers à délivrer les messages qui permettront de hausser le niveau de connaissances des populations. Nul besoin pour cela de moyens modernes, de télémédecine ou de vidéoconférences. Il faut de la bonne volonté et de la ténacité pour délivrer des messages pédagogiques essentiels à l'amélioration des pathologies digestives en zone rurale africaine. Il faut que le programme de construction de latrines dans les concessions soit généralisé dans ces zones rurales.

« Sans cesse sur le métier remettez votre ouvrage !». Les gastroentérologues du continent africain doivent participer à ces actions d'enseignement et de formation et ne pas se cantonner à leur activité clinique et technique.

La santé publique ne peut s'améliorer qu'avec la participation efficace et désintéressée de tous les acteurs du monde médical. 